\title{
Improved semidefinite programming bounds for quadratic assignment problems with suitable symmetry
}

\author{
Etienne de Klerk • Renata Sotirov
}

Received: 15 September 2009 / Accepted: 1 September 2010 / Published online: 24 September 2010 C The Author(s) 2010. This article is published with open access at Springerlink.com

\begin{abstract}
Semidefinite programming (SDP) bounds for the quadratic assignment problem (QAP) were introduced in Zhao et al. (J Comb Optim 2:71-109, 1998). Empirically, these bounds are often quite good in practice, but computationally demanding, even for relatively small instances. For QAP instances where the data matrices have large automorphism groups, these bounds can be computed more efficiently, as was shown in Klerk and Sotirov (Math Program A, 122(2), 225-246, 2010). Continuing in the same vein, we show how one may obtain stronger bounds for QAP instances where one of the data matrices has a transitive automorphism group. To illustrate our approach, we compute improved lower bounds for several instances from the QAP library QAPLIB.
\end{abstract}

Keywords Quadratic assignment problem - Semidefinite programming · Group symmetry $\cdot$ Branch and bound

Mathematics Subject Classification (2000) $\quad$ 90C22 $\cdot$ 20Cxx $\cdot 70-08$

\section{Introduction}

We study the quadratic assignment problem (QAP) in the following form:

$$
\min _{\pi \in \mathcal{S}_{n}} \sum_{i, j=1}^{n}\left(a_{i j} b_{\pi(i), \pi(j)}+c_{i, \pi(i)}\right),
$$

E. de Klerk $(\bowtie) \cdot$ R. Sotirov

Department of Econometrics and OR, Tilburg University, Tilburg, The Netherlands e-mail: e.deklerk@uvt.nl

R. Sotirov

e-mail: r.sotirov@uvt.nl 
where $A=\left[a_{i j}\right]$ and $B=\left[b_{i j}\right]$ are given symmetric $n \times n$ matrices, $C=\left[c_{i j}\right] \in \mathbb{R}^{n \times n}$, and $\mathcal{S}_{n}$ is the symmetric group on $n$ elements, i.e. the group of all permutations of $\{1, \ldots, n\}$. The matrices $A$ and $B$ are often called the distance and flow matrices respectively. The physical interpretation (when $C=0$ ) is that we are given $n$ facilities with specified flows between facilities given by the matrix $B$, as well as $n$ locations with relative distances between these locations given as the entries of $A$. The objective is to assign the facilities to locations such that the 'flow $\times$ distance' is minimal when summed over all pairs.

The QAP may be rewritten in terms of $n \times n$ permutation matrices as follows:

$$
\min _{X \in \Pi_{n}} \operatorname{tr}(A X B+C) X^{T}
$$

where $\Pi_{n}$ is the set of $n \times n$ permutation matrices. In this paper we will mostly restrict our attention to the case where $C=0$. We only need to deal with the linear term since it arises when doing branch and bound. To be precise, fixing a partial assignment of facilities to locations leads to a smaller QAP problem that always has a linear term, even if the original QAP does not; see Sect. 3.3.

The quadratic assignment problem is a well-known NP-hard problem and difficult to solve in practice for values $n \geq 30$; see [1] and the references therein.

Anstreicher et al. [1] recently achieved computational success in solving QAP instances by using nonlinear convex quadratic relaxations together with branch and bound.

Another class of convex relaxations for QAP are the semidefinite programming (SDP) bounds by Zhao et al. [25]. Empirically, these bounds are often quite good in practice, but computationally demanding for interior point solvers, even for relatively small instances (say $n \geq 15$ ). Lower order methods can solve the SDP relaxations for somewhat larger instances, but are known to be much slower than interior point methods; see Burer and Vandenbussche [3] for the state-of-the-art in lower order methods for these problems.

For QAP instances where the data matrices have large automorphism groups, the SDP bounds can be computed more efficiently, as was shown by De Klerk and Sotirov [10], who computed the SDP bound by Zhao et al. for some instances with $n$ up to 128 with interior point solvers.

Continuing in the same vein, we show how one may obtain stronger bounds for QAP instances where one of the data matrices has a transitive automorphism group. In particular, our approach is very suitable for QAP instances with Hamming distance matrices. To illustrate our approach, we compute improved lower bounds for several test problems from the QAP library QAPLIB [4].

\section{Notation}

The space of $p \times q$ matrices is denoted by $\mathbb{R}^{p \times q}$, the space of $k \times k$ symmetric matrices is denoted by $\mathbb{S}^{k \times k}$. For index sets $\alpha, \beta \subset\{1, \ldots, n\}$, we denote the submatrix that contains the rows of $A$ indexed by $\alpha$ and the columns indexed by $\beta$ as $A(\alpha, \beta)$. If $\alpha=\beta$, the principal submatrix $A(\alpha, \alpha)$ of $A$ is abbreviated as $A(\alpha)$. The $i$ th column of a matrix $C$ we denote by $C_{:, i}$. 
We use $I_{n}$ to denote the identity matrix of $\operatorname{order} n$, and $J_{n}$ the $n \times n$ all-ones matrix. We omit the subscript if the order is clear from the context. Also, $E_{i i}=e_{i} e_{i}^{T}$ where $e_{i}$ is the $i$-th standard basis vector.

The vec operator stacks the columns of a matrix, while the Diag operator maps an $n$-vector to an $n \times n$ diagonal matrix in the obvious way. The trace operator is denoted by 'tr'.

The Kronecker product $A \otimes B$ of matrices $A \in \mathbb{R}^{p \times q}$ and $B \in \mathbb{R}^{r \times s}$ is defined as the $p r \times q s$ matrix composed of $p q$ blocks of size $r \times s$, with block $i j$ given by $\left.a_{i j} B i=1, \ldots, p\right),(j=1, \ldots, q)$. The following properties of the Kronecker product will be used in the paper, see e.g. [13] (we assume that the dimensions of the matrices appearing in these identities are such that all expressions are well-defined):

$$
\begin{aligned}
(A \otimes B)^{T} & =A^{T} \otimes B^{T}, \\
(A \otimes B)(C \otimes D) & =A C \otimes B D .
\end{aligned}
$$

\section{SDP relaxation of QAP}

The following SDP relaxation of QAP was studied by Povh and Rendl [20]:

$$
\begin{array}{cl}
\min & \operatorname{tr}(B \otimes A+\operatorname{Diag}(\operatorname{vec}(C))) Y \\
\text { s.t. } & \operatorname{tr}\left(I_{n} \otimes E_{j j}\right) Y=1, \operatorname{tr}\left(E_{j j} \otimes I_{n}\right) Y=1 j=1, \ldots, n \\
& \operatorname{tr}\left(I_{n} \otimes\left(J_{n}-I_{n}\right)+\left(J_{n}-I_{n}\right) \otimes I_{n}\right) Y=0 \\
& \operatorname{tr}\left(J_{n^{2}} Y\right)=n^{2} \\
& Y \geq 0, \quad Y \succeq 0 .
\end{array}
$$

Note that here $Y \in \mathbb{S}^{n^{2} \times n^{2}}$. One may easily verify that (4) is indeed a relaxation of the QAP (1) by noting that a feasible point of (4) is given by

$$
Y:=\operatorname{vec}(X) \operatorname{vec}(X)^{T} \text { if } X \in \Pi_{n},
$$

and that the objective value of (4) at this point $Y$ is precisely $\operatorname{tr}(A X B+C) X^{T}$.

Povh and Rendl [20] showed that the relaxation (4) is equivalent to the earlier relaxation of Zhao et al. [25]. The latter relaxation is known to give good bounds in practice, but is difficult to solve with interior point methods for $n \geq 15$, due to its size. De Klerk and Sotirov [10] showed how to exploit algebraic symmetry of the matrices $A$ and $B$ when present, in order to reduce the computational effort of solving (4). In the next section we give a brief overview of this approach.

\section{Exploiting group symmetry in the SDP problems}

\subsection{General theory}

The discussion in this subsection is condensed from De Klerk and Sotirov [10]. More details may be found in the survey by Parrilo and Gatermann [12], or the thesis of Gijswijt [9]. 
Assume that the following semidefinite programming problem is given

$$
p^{*}:=\min _{X \succeq 0, X \geq 0}\left\{\operatorname{tr}\left(A_{0} X\right): \operatorname{tr}\left(A_{k} X\right)=b_{k}, \quad k=1, \ldots, m\right\}
$$

where $A_{i} \in \mathbb{S}^{n \times n}(i=0, \ldots, m)$ are given. We also assume that this problem has an optimal solution.

Assumption 1 (Group symmetry) We assume that there is a nontrivial multiplicative group of orthogonal matrices $\mathcal{G}$ such that the associated Reynolds operator

$$
R_{\mathcal{G}}(X):=\frac{1}{|\mathcal{G}|} \sum_{P \in \mathcal{G}} P^{T} X P, \quad X \in \mathbb{R}^{n \times n}
$$

maps the feasible set of (5) into itself and leaves the objective value invariant, i.e.

$$
\operatorname{tr}\left(A_{0} R(X)\right)=\operatorname{tr}\left(A_{0} X\right) \quad \text { if } X \text { is a feasible point of (5). }
$$

Since the Reynolds operator maps the convex feasible set into itself and preserves the objective values of feasible solutions, we may restrict the optimization to feasible points in the commutant (or centralizer ring) of $\mathcal{G}$, defined as:

$$
\begin{aligned}
\mathcal{A}_{\mathcal{G}}: & =\left\{X \in \mathbb{R}^{n \times n}: R_{\mathcal{G}}(X)=X\right\} \\
& =\left\{X \in \mathbb{R}^{n \times n}: X P=P X \quad \forall P \in \mathcal{G}\right\} .
\end{aligned}
$$

Note that $R_{\mathcal{G}}$ gives an orthogonal projection onto $\mathcal{A}_{\mathcal{G}}$.

The commutant $\mathcal{A}_{\mathcal{G}}$ is a matrix $*$-algebra over $\mathbb{R}$, i.e. a subspace of $\mathbb{R}^{n \times n}$ that is closed under matrix multiplication and taking transposes.

Orthonormal eigenvectors of the linear operator $R_{\mathcal{G}}$ corresponding to the eigenvalue 1 form an orthonormal basis of $\mathcal{A}_{\mathcal{G}}$ (seen as a vector space). This basis, say $\left.B_{1}, \ldots, B_{d} d:=\operatorname{dim}\left(\mathcal{A}_{\mathcal{G}}\right)\right)$, has the following properties:

- $B_{i} \in\{0,1\}^{n \times n}(i=1, \ldots, d)$;

$-\sum_{i=1}^{d} B_{i}=J$

- For any $i \in\{1, \ldots, d\}$, one has $B_{i}^{T}=B_{i *}$ for some $i * \in\{1, \ldots, d\}$ (possibly $i *=i)$.

One may also obtain the basis $B_{1}, \ldots, B_{d}$ from the orbitals of the group $\mathcal{A}_{\mathcal{G}}$. The orbital of the pair $(i, j)$ is defined as

$$
\left\{\left(P e_{i}, P e_{j}\right): P \in \mathcal{G}\right\}
$$

The corresponding basis matrix has an entry 1 at position $(k, l)$ if $\left(e_{k}, e_{l}\right)$ belongs to the orbital, and is zero otherwise.

The next result shows that one may replace the data matrices $A_{i}(i=0, \ldots, m)$ in the SDP formulation (5) by their projections $R\left(A_{i}\right)(i=0, \ldots, m)$ onto the commutant. 
Theorem 1 One has

$$
p^{*}=\min _{X \succeq 0, X \geq 0}\left\{\operatorname{tr}\left(R_{\mathcal{G}}\left(A_{0}\right) X\right): \operatorname{tr}\left(R_{\mathcal{G}}\left(A_{k}\right) X\right)=b_{k} \quad k=1, \ldots, m\right\}
$$

Proof The proof is an immediate consequence of Assumption 1 and the observation that $\operatorname{tr}\left(A_{i} R_{\mathcal{G}}(X)\right)=\operatorname{tr}\left(R_{\mathcal{G}}\left(A_{i}\right) X\right)$ for any $i$.

By Theorem 1, we may assume without loss of generality that there exists an optimal $X \in \mathcal{A}_{\mathcal{G}}$.

Assume we have a basis $B_{1}, \ldots, B_{d}$ of the commutant $\mathcal{A}_{\mathcal{G}}$. One may write $X=$ $\sum_{i=1}^{d} x_{i} B_{i}$. Moreover, the nonnegativity condition $X \geq 0$ is equivalent to $x \geq 0$, by the properties of the basis.

Thus the SDP problem (5) reduces to

$$
\min _{\sum_{i=1}^{d} x_{i} B_{i} \succeq 0, x \geq 0}\left\{\sum_{i=1}^{d} x_{i} \operatorname{tr}\left(R_{\mathcal{G}}\left(A_{0}\right) B_{i}\right): \sum_{i=1}^{d} x_{i} \operatorname{tr}\left(R_{\mathcal{G}}\left(A_{k}\right) B_{i}\right)=b_{k} \forall k\right\} .
$$

Note that the values $\operatorname{tr}\left(R_{\mathcal{G}}\left(A_{k}\right) B_{i}\right)(i=1, \ldots, d),(k=0, \ldots, m)$ may be computed beforehand.

The next step in reducing the SDP (6) is to block diagonalize the commutant $\mathcal{A}_{\mathcal{G}}$, i.e. block diagonalize its basis $B_{1}, \ldots, B_{d}$.

This is always possible due to a classical 'structure' theorem for matrix $*$-algebras. Before stating the result, recall that a matrix $*$-algebra $\mathcal{A}$ is called simple if its only ideals are $\{0\}$ and $\mathcal{A}$ itself.

Theorem 2 (Wedderburn [23]; see also [24]) Assume $\mathcal{A} \subset \mathbb{R}^{n \times n}$ is a matrix *-algebra over $\mathbb{R}$ that contains the identity I. Then there is an orthogonal matrix $Q$ and some integer s such that

$$
Q^{T} \mathcal{A} Q=\left(\begin{array}{cccc}
\mathcal{A}_{1} & 0 & \cdots & 0 \\
0 & \mathcal{A}_{2} & & \vdots \\
\vdots & & \ddots & 0 \\
0 & \cdots & 0 & \mathcal{A}_{s}
\end{array}\right)
$$

where each $\mathcal{A}_{t}(t=1, \ldots, s)$ is a simple matrix $*$-algebra over $\mathbb{R}$. This decomposition is unique up to the ordering of the blocks.

Simple matrix $*$-algebras over $\mathbb{R}$ are completely classified and one can give a more detailed statement of the above theorem. For our purposes, though, it suffices to note that block-diagonal structure may be exploited by interior point software, e.g. SeDuMi [22]. The final step in the symmetry reduction is therefore to replace the linear matrix inequality $\sum_{i=1}^{d} x_{i} B_{i} \succeq 0$ in (6) by the block diagonal equivalent: $\sum_{i=1}^{d} x_{i} Q^{T} B_{i} Q \succeq 0$, where $Q$ block-diagonalizes $\mathcal{A}_{\mathcal{G}}$ (cf. Theorem 2). Note that identical blocks appearing in the block diagonalization may be removed. 
3.2 The symmetry of the SDP relaxation of the QAP

We now apply the theory described in the last section to the SDP relaxation (4) of the QAP.

Note that, if $C=0$, the data matrices in (4) are

$B \otimes A, \quad E_{j j} \otimes I$ and $I \otimes E_{j j}(j=1, \ldots, n), J \otimes J$, and $(I \otimes(J-I)+(J-I) \otimes I)$.

Definition 3 We define the automorphism group of a matrix $Z \in \mathbb{R}^{n \times n}$ as

$$
\operatorname{aut}(Z)=\left\{P \in \Pi_{n}: P Z P^{T}=Z\right\}
$$

Theorem 4 Define the multiplicative matrix group

$$
\mathcal{G}_{Q A P}:=\left\{\left(P_{B} \otimes P_{A}\right): P_{A} \in \operatorname{aut}(A), P_{B} \in \operatorname{aut}(B)\right\}=: \mathcal{G}_{\text {aut }(B)} \otimes \mathcal{G}_{\text {aut }(A)} .
$$

Then the SDP problem (4) with $C=0$ satisfies Assumption 1 with respect to the group $\mathcal{G}_{Q A P}$.

Proof We have to show that the Reynolds operator $R_{\mathcal{G}_{Q A P}}$ maps the feasible set of (4) into itself and leaves the objective function invariant. Assume therefore that $Y \in$ $\mathbb{S}^{n^{2} \times n^{2}}$ is a feasible point of (4).

The objective value at $R_{\mathcal{G}_{Q A P}}(Y)$ is

$$
\begin{aligned}
\operatorname{tr} & \left((B \otimes A) R_{\mathcal{G}_{Q A P}}(Y)\right) \\
& =\operatorname{tr}\left(R_{\mathcal{G}_{Q A P}}(B \otimes A) Y\right) \\
& =\frac{1}{\left|\mathcal{G}_{Q A P}\right|} \sum_{P_{A} \in \operatorname{aut}(A), P_{B} \in \operatorname{aut}(B)} \operatorname{tr}\left(\left(P_{B} \otimes P_{A}\right)^{T}(B \otimes A)\left(P_{B} \otimes P_{A}\right) Y\right) \\
& =\frac{1}{\left|\mathcal{G}_{Q A P}\right|} \sum_{P_{A} \in \operatorname{aut}(A), P_{B} \in \operatorname{aut}(B)} \operatorname{tr}\left(\left(P_{B}^{T} B P_{B} \otimes P_{A}^{T} A P_{A}\right) Y\right) \\
& =\operatorname{tr}((B \otimes A) Y),
\end{aligned}
$$

where we have used the properties (2) and (3) of the Kronecker product.

In exactly the same way we may show that the following two constraints are satisfied:

$$
\begin{aligned}
\operatorname{tr}\left((I \otimes(J-I)+(J-I) \otimes I) R_{\mathcal{G}_{Q A P}}(Y)\right) & =0 \\
& \operatorname{tr}\left(J R_{\mathcal{G}_{Q A P}}(Y)\right)=n^{2}
\end{aligned}
$$

Since $R_{\mathcal{G}_{Q A P}}(Y) \succeq 0$ and $R_{\mathcal{G}_{Q A P}}(Y) \geq 0$ it only remains to show that

$$
\operatorname{tr}\left(I \otimes E_{j j}\right) R_{\mathcal{G}_{Q A P}}(Y)=1, \quad \operatorname{tr}\left(E_{j j} \otimes I\right) R_{\mathcal{G}_{Q A P}}(Y)=1 \quad j=1, \ldots, n
$$


To this end, we fix $j \in\{1, \ldots, n\}$. One has:

$$
\begin{aligned}
\operatorname{tr} & \left(I \otimes E_{j j}\right) R_{\mathcal{G}_{Q A P}}(Y) \\
& =\operatorname{tr}\left(R_{\mathcal{G}_{Q A P}}\left(I \otimes E_{j j}\right) Y\right) \\
& =\frac{1}{\left|\mathcal{G}_{Q A P}\right|} \sum_{P_{A} \in \operatorname{aut}(A), P_{B} \in \operatorname{aut}(B)} \operatorname{tr}\left(\left(P_{B} \otimes P_{A}\right)^{T}\left(I \otimes E_{j j}\right)\left(P_{B} \otimes P_{A}\right) Y\right) \\
& =\frac{1}{\left|\mathcal{G}_{Q A P}\right|} \sum_{P_{A} \in \operatorname{aut}(A), P_{B} \in \operatorname{aut}(B)} \operatorname{tr}\left(\left(I \otimes P_{A}^{T} E_{j j} P_{A}\right) Y\right) \\
& =\frac{1}{\left|\mathcal{G}_{Q A P}\right|} \sum_{P_{A} \in \operatorname{aut}(A), P_{B} \in \operatorname{aut}(B)} 1=1,
\end{aligned}
$$

where we have again used the properties (2) and (3) of the Kronecker product as well as $\operatorname{tr}\left(I \otimes E_{k k}\right) Y=1$ for all $k=1, \ldots, n$. The proof of $\operatorname{tr}\left(E_{j j} \otimes I\right) R_{\mathcal{G}_{Q A P}}(Y)=1$ proceeds in the same way.

One may also construct the commutant of $\mathcal{G}_{Q A P}$ form the commutants of aut $(A)$ and $\operatorname{aut}(B)$ as follows.

Theorem 5 ( $c f$. Theorem 6.2 in [10]) Let $\mathcal{A}_{\text {aut }(A)}$ denote the commutant of aut $(A)$, etc. One has:

$$
\mathcal{A}_{\mathcal{G}_{Q A P}}=\mathcal{A}_{\mathrm{aut}(B)} \otimes \mathcal{A}_{\mathrm{aut}(A)}:=\left\{X_{B} \otimes X_{A}: X_{A} \in \mathcal{A}_{\mathrm{aut}(A)}, X_{B} \in \mathcal{A}_{\mathrm{aut}(B)}\right\}
$$

The general theory of symmetry reduction may therefore be applied to the SDP problem (4) in a mechanical way; this was done in detail in [10]. In what follows we are interested in a different SDP relaxation, that is derived by considering a partial assignment (assigning one facility to a location). Equivalently, we will consider relaxations in the first level of the branching tree for QAP.

\subsection{Symmetry reduction in the first level of the branching tree}

In the following lemma, we show that when we fix some entry in the permutation matrix $X$ to 1 , we obtain a QAP problem that is one dimension smaller than the original one. In terms of the physical interpretation of the QAP, we are assigning facility $s$ to location $r$ for a given index pair $(r, s)$. In terms of branch and bound, we are considering a child node at the first level of the branching tree.

Lemma 6 Let $X \in \Pi_{n}$, and $r, s \in\{1, \ldots, n\}$ such that $X_{r, s}=1$. Then for $\alpha=$ $\{1, \ldots, n\} \backslash r$ and $\beta=\{1, \ldots, n\} \backslash$ s one has

$$
\operatorname{tr}(A X B+C) X^{T}=\operatorname{tr}(A(\alpha) X(\alpha, \beta) B(\beta)+\bar{C}(\alpha, \beta)) X(\alpha, \beta)^{T}+d,
$$

where 


$$
\bar{C}(\alpha, \beta)=C(\alpha, \beta)+2 A(\alpha,\{r\}) B(\{s\}, \beta)
$$

and $d=a_{r, r} b_{s, s}+c_{r, s}$.

Proof By fixing $X_{r s}=1$, we get

$$
\begin{aligned}
\operatorname{tr}\left(A X B X^{T}\right)= & \sum_{i, k=1}^{n}(A X B)_{i k} x_{i k}=\sum_{i, k, j, l=1}^{n} a_{i j} x_{j l} b_{k l} x_{i k} \\
= & \sum_{i \neq r, k \neq s, j \neq r, l \neq s}^{n} a_{i j} x_{j l} b_{k l} x_{i k}+\sum_{i \neq r, k \neq s}^{n} a_{i r} b_{k s} x_{i k} \\
& +\sum_{j \neq r, l \neq s}^{n} a_{r j} b_{s l} x_{j l}+a_{r r} b_{s s} \\
= & \sum_{i \neq r, k \neq s, j \neq r, l \neq s}^{n} a_{i j} x_{j l} b_{k l} x_{i k}+2 \sum_{i \neq r, k \neq s}^{n} a_{i r} b_{k s} x_{i k}+a_{r r} b_{s s} .
\end{aligned}
$$

Moreover,

$$
\operatorname{tr}\left(C X^{T}\right)=\sum_{i, k=1}^{n} c_{i k} x_{i k}=\sum_{i \neq r, k \neq s}^{n} c_{i k} x_{i k}+c_{r s}
$$

which proves the lemma.

Since $A(\alpha), B(\beta) \in \mathbb{S}^{n-1 \times n-1}$ and $X(\alpha, \beta) \in \Pi_{n-1}$, the reduced problem

$$
\min _{X \in \Pi_{n-1}} \operatorname{tr}(A(\alpha) X B(\beta)+\bar{C}(\alpha, \beta)) X^{T}
$$

is also a quadratic assignment problem, and its SDP relaxation (4) becomes

$$
\begin{array}{cl}
\min & \operatorname{tr}(B(\beta) \otimes A(\alpha)+\operatorname{Diag}(\bar{c})) Y \\
\text { s.t. } & \operatorname{tr}\left(I \otimes E_{j j}\right) Y=1, \operatorname{tr}\left(E_{j j} \otimes I\right) Y=1 \quad \forall j \\
& \operatorname{tr}(I \otimes(J-I)+(J-I) \otimes I) Y=0 \\
& \operatorname{tr}((J \otimes J) Y)=(n-1)^{2} \\
& Y \geq 0, \quad Y \succeq 0,
\end{array}
$$

where $I, J, E_{j j} \in \mathbb{R}^{(n-1) \times(n-1)}, \bar{c}=\operatorname{vec}(\bar{C}(\alpha, \beta))$, and $Y \in \mathbb{S}^{(n-1)^{2} \times(n-1)^{2}}$.

Note that the data matrices of the SDP problem (10) are

$$
B(\beta) \otimes A(\alpha)+\operatorname{Diag}(\bar{c}), J \otimes J,(I \otimes(J-I))+(J-I) \otimes I), E_{j j} \otimes I, I \otimes E_{j j},
$$

where $j=1, \ldots, n-1$ and all matrices are of order $n-1$. 
In order to perform the symmetry reduction of the SDP (10), we therefore need to find the automorphism groups of the matrices in (11). To this end, we need one more definition.

Definition 7 For fixed $r \in\{1, \ldots, n\}$, the subgroup of aut $(A)$ that fixes $r$ is:

$$
\operatorname{stab}(r, A):=\left\{P \in \operatorname{aut}(A): P_{r, r}=1\right\}
$$

This is known as the stabilizer subgroup of aut $(A)$ with respect to $r$; see e.g., $[5,9]$.

The importance of the stabilizer group becomes clear from the following lemma.

Lemma 8 For $\alpha=\{1, \ldots, n\} \backslash r$ the automorphism group of $A(\alpha)$ is given by

$$
\operatorname{aut}(A(\alpha))=\{P(\alpha): P \in \operatorname{stab}(r, A)\} .
$$

Proof Follows directly from the definition of stabilizer (12).

Thus we may readily obtain $\operatorname{aut}(A(\alpha))$ from $\operatorname{stab}(r, A)$.

The next lemma will be used to describe the automorphism group of the matrix $\operatorname{Diag}(\bar{c})$ in $(11)$.

Lemma 9 Let $A_{:, r}$ be the rth column of the matrix A. Then,

$$
P^{T} \operatorname{Diag}\left(A_{:, r}\right) P=\operatorname{Diag}\left(A_{:, r}\right), \quad \forall P \in \operatorname{stab}(r, A) .
$$

Proof For standard unit vectors $e_{r}, e_{k} \in \mathbb{R}^{n}$ and $P \in \operatorname{stab}(r, A)$ we have

$$
P^{T} e_{k} e_{r}^{T} P=e_{k^{\prime}} e_{r}^{T} \quad \text { and } \quad P^{T} e_{r} e_{k} P=e_{r} e_{k^{\prime}}^{T}
$$

for some $k^{\prime} \in\{1, \ldots, n\}$. Since $P \in \operatorname{aut}(A)$ it follows that $a_{k r}=a_{k^{\prime}}$ for all $\left(k^{\prime}, r\right)$ such that $\left(e_{k^{\prime}}, e_{r}\right)$ belongs to the same orbital as $\left(e_{k}, e_{r}\right)$. Now from

$$
P^{T} e_{k} e_{k}^{T} P=e_{k^{\prime}} e_{k^{\prime}}^{T}
$$

follows the proof of the lemma.

Finally, we derive the automorphism group of the matrix $B(\beta) \otimes A(\alpha)+\operatorname{Diag}(\bar{c})$ in (11).

Theorem 10 Let $r, s \in\{1, \ldots, n\}, \alpha=\{1, \ldots, n\} \backslash r, \beta=\{1, \ldots, n\} \backslash s$. If $C=0$, then

$$
\text { aut }(B(\beta) \otimes A(\alpha)+\operatorname{Diag}(\bar{c}))=\operatorname{aut}(B(\beta) \otimes A(\alpha))=\operatorname{aut}(B(\beta)) \otimes \operatorname{aut}(A(\alpha))
$$

where $\bar{c}=\operatorname{vec}(\bar{C}(\alpha, \beta))$. 
Proof If $C=0$, then $\bar{C}(\alpha, \beta)=2 A(\alpha,\{r\}) B(\{s\}, \beta)$ and $\operatorname{vec}(\bar{C})=2 B(\beta,\{s\}) \otimes$ $A(\alpha,\{r\})$. Now, from Lemma 9 and for $P_{B} \in \operatorname{aut}(B(\beta)), P_{A} \in \operatorname{aut}(A(\alpha))$ one has

$$
\begin{aligned}
& \left(P_{B} \otimes P_{A}\right)^{T} \operatorname{Diag}(B(\beta,\{s\}) \otimes A(\alpha,\{r\}))\left(P_{B} \otimes P_{A}\right) \\
= & \left(P_{B} \otimes P_{A}\right)^{T}(\operatorname{Diag}(B(\beta,\{s\})) \otimes \operatorname{Diag}(A(\alpha,\{r\})))\left(P_{B} \otimes P_{A}\right) \\
= & P_{B}^{T} \operatorname{Diag}(B(\beta,\{s\})) P_{B} \otimes P_{A}^{T} \operatorname{Diag}(A(\alpha,\{r\})) P_{A} \\
= & \operatorname{Diag}(B(\beta,\{s\}) \otimes A(\alpha,\{r\})),
\end{aligned}
$$

where we have used the properties (2) and (3) of the Kronecker product.

We are now in a position to formally describe in which sense the SDP (10) meets Assumption 1 (the symmetry assumption).

Theorem 11 The SDP problem (10) satisfies Assumption 1 with respect to the group $\mathcal{G}_{r, s}:=\operatorname{aut}(B(\beta)) \otimes \operatorname{aut}(A(\alpha))$.

Proof The proof is similar to that of Theorem 4 and is therefore omitted.

Thus we may again proceed in a mechanical way to perform the symmetry reduction of the SDP problem (10). In particular, we may restrict the variable $Y$ in (10) to lie in the commutant

$$
\mathcal{A}_{\mathcal{G}_{r, s}}=\mathcal{A}_{\mathrm{aut}(B(\beta))} \otimes \mathcal{A}_{\mathrm{aut}(A(\alpha))},
$$

and we may obtain a basis of this algebra from the orbitals of aut $(B(\beta))$ and aut $(A(\alpha))$, as before.

\section{Bounds if $\operatorname{aut}(A)$ or $\operatorname{aut}(B)$ is transitive}

In the last section we showed how to obtain lower bounds at the first level of the branching tree, i.e. by solving the SDP (10). In general, these bounds are not lower bounds for the original QAP problem, but if aut $(A)$ or aut $(B)$ is transitive, we do obtain such global lower bounds as the next lemma shows.

Lemma 12 If aut (A) or aut $(B)$ is transitive and $C=0$, then any lower bound for the QAP subproblem (9) at the first level of the branching tree is also a lower bound for the original $Q A P$.

Proof Assume aut $(B)$ is transitive and consider the QAP in the combinatorial formulation

$$
\min _{\pi \in \mathcal{S}_{n}} \sum_{i, j=1}^{n} a_{i j} b_{\pi(i), \pi(j)} .
$$

Let $\pi^{\prime}$ denote the optimal permutation. Note that

$$
\sum_{i, j=1}^{n} a_{i j} b_{\sigma\left(\pi^{\prime}(i)\right), \sigma\left(\pi^{\prime}(j)\right)}
$$


is also an optimal solution of the QAP for any $\sigma$ in aut(B). Let $r, s \in\{1, \ldots, n\}$ be given. There exists a $\sigma \in \operatorname{aut}(\mathrm{B})$ such that $\sigma\left(\pi^{\prime}(r)\right)=s$, since aut(B) is transitive.

Letting $\pi^{*}:=\sigma \pi^{\prime}$ one has $\pi^{*}$ is an optimal permutation for the QAP and $\pi^{*}(r)=$ $s$. This means that, for any given $r, s$ there is an optimal assignment that assigns facility $r$ to location $s$.

Thus every child node at the first level yields a lower bound on the global min of the QAP.

If one of the automorphism groups of the data matrices is transitive, say aut $(B)$, then the number of different subproblems in the first level of the branching tree depends on the number of orbits of aut $(A)$. We give the details in the following lemma.

Lemma 13 Let aut $(B)$ be transitive, then there are as many different child subproblems at the first level of the branching tree as there are orbits of aut $(A)$.

Proof Suppose that $e_{i}, e_{k}$ belong to the same orbit of aut $(A)$. Thus, there is a $P \in$ $\operatorname{aut}(A)$ for which $P e_{i}=e_{k}$.

Let $X$ be a solution of the child problem where $X_{i s}=1$. Since $A=P^{T} A P$, it follows

$$
\operatorname{tr}\left(A X B X^{T}\right)=\operatorname{tr}\left(P^{T} A P X B X^{T}\right)=\operatorname{tr}\left(A(P X) B(P X)^{T}\right) .
$$

Thus, $\bar{X}=P X$ is a feasible solution for the subproblem for which $\bar{X}_{k s}=1$, and with the same value of the objective function as for the subproblem with $X_{i s}=1$.

The results of the last two lemmas are undoubtedly known, if not exactly in this form. A detailed treatment on exploiting group symmetry in branch and bound trees may be found in [18].

\section{Example: the Terwilliger algebra of the Hamming scheme}

In Sect. 6, we will present computational results for QAP instances where one of the data matrix is a Hamming distance matrix. (Several QAP instances from QAPLIB [4] have such data matrices, namely the 'esc' instances [8]).

In this section we therefore review the details of the relevant algebraic symmetry; our presentation is condensed from the thesis of Gijswijt [9].

Consider the matrix $A$ with $2^{d}$ rows indexed by all elements of $\{0,1\}^{d}$, and $A_{i j}$ given by the Hamming distance between $i \in\{0,1\}^{d}$ and $j \in\{0,1\}^{d}$.

The automorphism group of $A$ arises as follows. Any permutation $\pi$ of the index set $\{1, \ldots, d\}$ induces an isomorphism of $A$ that maps row (resp. column) $i$ of $A$ to row (resp.column) $\pi(i)$ for all $i$. There are $d$ ! such permutations. Moreover, there are an additional $2^{d}$ permutations that act on $\{0,1\}^{d}$ by either 'flipping' a given component from zero to one (and vice versa), or not.

Thus aut $(A)$ has order $d ! 2^{d}$ and is transitive. The centralizer $\operatorname{ring}$ of $\operatorname{aut}(A)$ is a commutative matrix $*$-algebra over $\mathbb{R}$ and is known as the Bose-Mesner algebra of the Hamming scheme. 
Now fix some $u \in\{0,1\}^{d}$, and consider the stabilizer subgroup of aut $(A)$ with respect to $u$.

The orbital matrices of this stabilizer group look as follows:

$$
\left(M_{i, j}^{t}\right)_{v, w}= \begin{cases}1 & \text { if } d(u, v)=i, d(u, w)=j \text { and } t=\left|\left\{k \mid u_{k} \neq v_{k}=w_{k}\right\}\right| \\ 0 & \text { otherwise. }\end{cases}
$$

Here $d(u, v)$ is the Hamming distance between $u$ and $v$, etc, and $i, j, t$ take the values $0,1, \ldots, d$. There are $\left(\begin{array}{c}d+3 \\ 3\end{array}\right)$ such orbital matrices. Note that we may assume w.l.o.g. that $u=0$, since we may label the rows and columns arbitrarily. Then the above definition of $M_{i, j}^{t}$ becomes:

$$
\left(M_{i, j}^{t}\right)_{v, w}= \begin{cases}1 & \text { if }|S(v)|=i,|S(w)|=j \text { and } t=|S(v) \cap S(w)| \\ 0 & \text { otherwise, }\end{cases}
$$

where $S(v)$ is the support of $v$, i.e. the set of indices $\left\{i: v_{i}=1\right\}$.

We now describe the blocks in the matrix

$$
\widetilde{M_{i, j}^{t}}:=Q^{T} M_{i, j}^{t} Q
$$

where $Q$ is the orthogonal matrix that block diagonalizes the Terwilliger algebra of the Hamming scheme (cf. Theorem 2). The full details of this block diagonalization were first derived by Schrijver [21].

Each matrix $\widetilde{M_{i, j}^{t}}$ has $\lfloor d / 2\rfloor+1$ blocks (when ignoring multiplicities of the blocks), and the block sizes are

$$
d+1, \quad d-1, d-3, \ldots
$$

We will index the blocks by $k=0, \ldots,\lfloor d / 2\rfloor$, so that block $k$ has size $(d+1-2 k) \times$ $(d+1-2 k)$ and multiplicity $\left(\begin{array}{l}d \\ k\end{array}\right)-\left(\begin{array}{c}d \\ k-1\end{array}\right)$.

Block $k$ of $\widetilde{M_{i, j}^{t}}$ has at most one nonzero entry. It has one nonzero entry if $i, j \in$ $\{k, k+1, \ldots, d-k\}$, and then the entry takes the value:

$$
\left(\begin{array}{c}
d-2 k \\
i-k
\end{array}\right)^{-\frac{1}{2}}\left(\begin{array}{c}
d-2 k \\
j-k
\end{array}\right)^{-\frac{1}{2}} \beta_{i, j, k}^{t}
$$

where

$$
\beta_{i, j, k}^{t}=\left(\begin{array}{c}
d-2 k \\
i-k
\end{array}\right) \sum_{p=0}^{d}(-1)^{k-p}\left(\begin{array}{l}
k \\
p
\end{array}\right)\left(\begin{array}{c}
i-p \\
t-p
\end{array}\right)\left(\begin{array}{c}
d+p-i-k \\
d+t-i-j
\end{array}\right)
$$

An alternative, equivalent definition of $\beta_{i, j, k}^{t}$ is given in (3.57) on page 30 of [9]. The nonzero entry is in position $(i-k+1, j-k+1)$ in the block. 
Table 1 Dimension of the commutant for esc instances

\begin{tabular}{lccr}
\hline Instance & $\operatorname{dim} \mathcal{A}_{\mathcal{G}_{Q A P}} \cap \mathbb{S}^{n^{2} \times n^{2}}$ & $\operatorname{dim} \mathcal{A}_{\mathcal{G}_{r s}} \cap \mathbb{S}^{(n-1)^{2} \times(n-1)^{2}}$ & $\operatorname{dim} \mathbb{S}^{n^{2} \times n^{2}}$ \\
\hline esc32a & 1,656 & 13,153 & 524,800 \\
esc32b & 72 & 6,207 & 524,800 \\
esc32c & 265 & 1,988 & 524,800 \\
esc32d & 249 & 2,479 & 524,800 \\
esc32h & 499 & 3,848 & 524,800 \\
esc64a & 517 & 6,110 & $8,390,656$ \\
\hline
\end{tabular}

Thus, after removing the repeated blocks from $\widetilde{M_{i, j}^{t}}$, one obtains a block diagonal matrix, say:

$$
\left(\begin{array}{llll}
B_{0}^{(i, j, t)} & & & \\
& B_{1}^{(i, j, t)} & & \\
& & \ddots & \\
& & & B_{\lfloor d / 2\rfloor}^{(i, j, t)}
\end{array}\right)
$$

where $B_{k}^{(i, j, t)} \in \mathbb{R}^{(d+1-2 k) \times(d+1-2 k)}(k=0, \ldots,\lfloor d / 2\rfloor)$, and $B_{k}^{(i, j, t)}$ has at most one nonzero element given by:

$$
\begin{aligned}
& {\left[B_{k}^{(i, j, t)}\right]_{i-k+1, j-k+1}} \\
& \quad=\left\{\begin{array}{l}
\left(\begin{array}{c}
d-2 k \\
i-k
\end{array}\right)^{-\frac{1}{2}}\left(\begin{array}{c}
d-2 k \\
j-k
\end{array}\right)^{-\frac{1}{2}} \beta_{i, j, k}^{t} \quad \text { if } i, j \in\{k, k+1, \ldots, d-k\} \\
0 \text { otherwise. }
\end{array}\right.
\end{aligned}
$$

\section{Numerical results}

In Table 1 we list dimensions of the commutants $\mathcal{A}_{\mathcal{G}_{Q A P}}$ in (8) and $\mathcal{A}_{\mathcal{G}_{r, s}}$ in (13) for the esc instances [8] in the QAPlib library [4]. Recall that these dimensions determine the sizes of the SDP relaxations (4) and (10) respectively. We also list the dimension of $\mathbb{S}^{n^{2} \times n^{2}}$ in the table, to show how much the problem size is reduced.

The value $n$ for these instances can be read from the name of the instance. The distance matrix $A$ for these instances has the algebraic symmetry described in Sect. 5, i.e. it is a Hamming distance matrix.

In Table 2 we list the number of different child nodes at the first level of the branching tree for each instance ( $c f$. Lemma 13).

In Table 3 we list the new SDP lower bounds (10) that we computed using symmetry reduction. The previous best lower bounds are also listed together with the literature reference where the bounds were reported. 
Table 2 Number of different child nodes for the esc instances

Table 3 Bounds and solution times for the larger esc instances

l.b. lower bound, $u . b$. upper bound

\begin{tabular}{lc}
\hline Instance & $\sharp$ of child nodes \\
\hline esc32a & 26 \\
esc32b & 2 \\
esc32c & 10 \\
esc32d & 9 \\
esc32h & 14 \\
esc64a & 13 \\
\hline
\end{tabular}

Instance Previous 1.b. (4) SDP l.b. (10) Best known u.b. Time(s)

\begin{tabular}{llllr}
\hline esc32a & $104([10])$ & 107 & 130 & 191,510 \\
esc32b & $132([3])$ & 141 & 168 & 21,234 \\
esc32c & $616([3])$ & 618 & 642 & 256 \\
esc32d & $191([3])$ & 194 & 200 & 132 \\
esc32h & $425([10])$ & 427 & 438 & 1,313 \\
esc64a & $98([10])$ & 105 & 116 & 24,275 \\
\hline
\end{tabular}

It is clear from Table 3 that the new SDP bound (10) can be significantly better than the SDP bound (4). In fact, improved lower bounds are obtained for all the instances in the table.

The stronger bounds are obtained at a significant computational cost, though, as may be seen from the solution times listed in Table 3. The bounds for esc $32 a$, esc $32 b$ and esc $64 a$ were computed by SDPA-DD solver ${ }^{1}$ (private communication with Katsuki Fujisawa), since these problems showed poor numerical conditioning. The high running times for these instances reflect the fact that SDPA-DD uses high precision computations. All other bounds were done with SeDuMi [22] using the Yalmip interface [16] on a Pentium IV 3.4 GHz dual-core processor.

For problem esc 128 in the QAPlib library, there are 16384 orbitals for stab $(1, A)$. Although we are able to compute them, we were not able to form the problem itself. We note that it is possible to solve the SDP relaxation (4) for this instance using symmetry reduction; see [10].

\section{Concluding remarks}

The approach in this paper has two potential areas of application, that we discuss here in more detail.

7.1 QAP's with Hamming distance matrices

QAP problems with Hamming distance matrices arise in several applications:

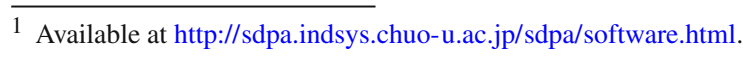


- The esc instances in the QAPlib arise from the design of hardwired VLSI control units [8]; see also the survey [7].

- In information theory, there are applications in channel coding; see [2] and [19].

- Harper [14] considered the problem of assigning the numbers $1, \ldots, 2^{d}$ to the vertices of the $0-1$ hypercube in $d$ dimensions so as to minimize

$$
\sum_{i, j=1}^{2^{d}} a_{i j}|\pi(i)-\pi(j)|
$$

where $\pi(i)$ is the number assigned to vertex $i\left(i=1, \ldots, 2^{d}\right)$, and $A=\left[a_{i j}\right]$ is the adjacency matrix of the hypercube. This is clearly a QAP where the matrix $A$ is a Hamming distance matrix and $b_{i j}:=|\pi(i)-\pi(j)|$. We should mention though, that a simple algorithm for this specific problem is given in [14] — it need not be solved as a QAP. Some variations of this problem remain unsolved, though. One such example that also leads to a QAP is where

$$
b_{i j}:=|\pi(i)-\pi(j)|^{s}
$$

for some given integer $s \geq 2$; see page 135 in [14].

In a recent paper, Mittelmann and Peng [17] give a detailed review of these and other QAP problems that involve Hamming distance matrices.

For problems of this type, we are therefore able to compute the SDP lower bound (10) for sizes up to $n=64$.

\subsection{QAP's where aut $(A)$ is transitive}

The second aspect of the research in this paper is that we obtained a new SDP bound for all QAP instances where the automorphism group of one of the data matrices is transitive.

One famous example of such an instance is the QAP reformulation of the traveling salesman problems (TSP). Indeed, defining

$$
A:=\frac{1}{2}\left[\begin{array}{cccccc}
0 & 1 & 0 & \cdots & 0 & 1 \\
1 & 0 & 1 & 0 & \cdots & 0 \\
0 & 1 & 0 & 1 & \ddots & \vdots \\
\vdots & \ddots & \ddots & \ddots & \ddots & \\
0 & & & & 0 & 1 \\
1 & 0 & \cdots & 0 & 1 & 0
\end{array}\right],
$$

and $B$ as the matrix of distances between cities, and $C=0$, the QAP (1) reduces to the TSP problem. The automorphism group of $A$ is the dihedral group in this case, which is transitive. Thus (10) provides a lower bound on the optimal value of the TSP instance, that is as least as tight as the bound (4). The SDP relaxation (4) may be 
Table 4 Lower bounds for TSP instances on 8 cities

\begin{tabular}{|c|c|c|c|c|}
\hline & $\begin{array}{l}\text { SDP } \\
\text { bound (4) }\end{array}$ & $\begin{array}{l}\text { Held-Karp } \\
\text { bound }\end{array}$ & $\begin{array}{l}\text { New SDP } \\
\text { bound }(10)\end{array}$ & $\begin{array}{l}\text { Optimal } \\
\text { value }\end{array}$ \\
\hline 1 & 2 & 2 & 2 & 2 \\
\hline 2 & 1.628 & 2 & 2 & 2 \\
\hline 3 & 1.172 & 2 & 1.893 & 2 \\
\hline 4 & 8.671 & 9 & 10 & 10 \\
\hline 5 & 9 & 9 & 10 & 10 \\
\hline 6 & 8.926 & 9 & 10 & 10 \\
\hline 7 & 8.586 & 9 & 10 & 10 \\
\hline 8 & 8.926 & 9 & 10 & 10 \\
\hline 9 & 9 & 9 & 10 & 10 \\
\hline 10 & 8.902 & 9 & 10 & 10 \\
\hline 11 & 8.899 & 9 & 10 & 10 \\
\hline 12 & 0 & 0 & 0 & 0 \\
\hline 13 & 10.667 & 11 & 11.777 & 12 \\
\hline 14 & 12 & 12 & 12.777 & 13 \\
\hline 15 & 12.444 & $12 \frac{2}{3}$ & 13.663 & 14 \\
\hline 16 & 14.078 & 14 & 15.651 & 16 \\
\hline 17 & 16 & 16 & 17.824 & 18 \\
\hline 18 & 16 & 16 & 17.698 & 18 \\
\hline 19 & 16 & 16 & 18 & 18 \\
\hline 20 & 15.926 & 16 & 18 & 18 \\
\hline 21 & 18.025 & 18 & 19.568 & 20 \\
\hline 22 & 20 & 20 & 21.287 & 22 \\
\hline 23 & 23.033 & 23 & 25.460 & 26 \\
\hline 24 & 34.739 & 35 & 37.141 & 38 \\
\hline
\end{tabular}

simplified for TSP, and the TSP lower bound it provides is known to be independent of the Held-Karp [15] bound; see [11] for details.

In Table 4 we show some computational results for TSP instances on 8 cities, constructed from the 24 classes of facet defining inequalities for the TSP polytope on 8 cities (described in [6]).

For the problems in Table 4, the new SDP bound (10) is better than the Held-Karp [15] bound, except for instance 3. Moreover, 13 of the 24 classes of facet defining inequalities are implied by the new relaxation (see Table 4). Since the data for these problems is integer, the bounds in the table may be rounded up. After doing so, the optimal value of the TSP is obtained in each case from the new SDP bound (10).

On the other hand, the new bound for TSP is computationally very intensive compared to the Held-Karp bound, and will be mainly of theoretical interest. Having said that, it is clear from the table that the new bound is strong, and it is a topic for future research to investigate its theoretical properties.

Acknowledgments The authors would like to thank Katsuki Fujisawa for solving several SDP instances for them using the SDPA-DD software. 
Open Access This article is distributed under the terms of the Creative Commons Attribution Noncommercial License which permits any noncommercial use, distribution, and reproduction in any medium, provided the original author(s) and source are credited.

\section{References}

1. Anstreicher, K.M.: Recent advances in the solution of quadratic assignment problems. Math. Program. Ser. B 97, 27-42 (2003)

2. Ben-David, G., Malah, D.: Bounds on the performance of vector quantizers under channel errors. IEEE Trans. Inf. Theory 51(6), 2227-2235 (2005)

3. Burer, S., Vandenbussche, D.: Solving lift-and-project relaxations of binary integer programs. SIAM J. Optim. 16, 726-750 (2006)

4. Burkard, R.E., Karisch, S.E., Rendl, F.: QAPLIB—a quadratic assignment problem library. J. Glob. Optim. 10, 291-403 (1997). see also http://www.seas.upenn.edu/qaplib/

5. Cameron, P.J.: Permutation Groups. Cambridge University Press, Cambridge (1999)

6. Christof, T., Jünger, M., Reinelt, G.: A complete description of the traveling salesman polytope on 8 nodes. Oper. Res. Lett. 10, 497-500 (1991)

7. Eschermann, B.: State assignment for hardwired VLSI control units. ACM Comput. Surv. 25(4), 415-436 (1993)

8. Eschermann, B., Wunderlich, H.J.: Optimized synthesis of self-testable finite state machines. In: 20th international symposium on fault-tolerant computing (FFTCS 20), Newcastle upon Tyne, 26-28th June (1990)

9. Gijswijt, D.: Matrix algebras and semidefinite programmming techniques for Codes. PhD Thesis. University of Amsterdam, The Netherlands (2005)

10. de Klerk, E., Sotirov, R.: Exploiting group symmetry in semidefinite programming relaxations of the quadratic assignment problem. Math. Program. A 122(2), 225-246 (2010)

11. de Klerk, E., Pasechnik, D.V., Sotirov, R.: On semidefinite programming relaxations of the traveling salesman problem. SIAM J. Optim. 19(4), 1559-1573 (2008)

12. Gatermann, K., Parrilo, P.A.: Symmetry groups, semidefinite programs, and sum of squares. J. Pure Appl. Algebra 192, 95-128 (2004)

13. Graham, A.: Kronecker products and matrix calculus with applications. Ellis Horwood Limited, Chichester (1981)

14. Harper, L.H.: Optimal assignments of numbers to vertices. SIAM J. Sci. Ind. Appl. Math. 12(1), 131-135 (1964)

15. Held, M., Karp, R.M.: The traveling salesman problem and minimum spanning trees. Oper. Res. 18, 1138-1162 (1970)

16. Löfberg, J.: YALMIP : A Toolbox for Modeling and Optimization in MATLAB. In: Proceedings of the CACSD conference, Taipei, Taiwan (2004). http://control.ee.ethz.ch/ joloef/yalmip.php

17. Mittelmann, H., Peng, J.: Estimating bounds for quadratic assignment problems associated with hamming and manhattan distance matrices based on semidefinite programming. SIAM J. Optim. (submitted, 2008)

18. Ostrowski, J., Linderoth, J., Rossi, F., Smiriglio, S.: Orbital branching. Math. Program. A (accepted 2009)

19. Potter, L.C., Chiang, D.M.: Minimax nonredundant channel coding. IEEE Trans. Commun. 43(2/3/4), 804-811 (1995)

20. Povh, J., Rendl, F.: Copositive and semidefinite relaxations of the quadratic assignment problem. Discrete Optim. 6(3), 231-241 (2009)

21. Schrijver, A.: New code upper bounds from the Terwilliger algebra. IEEE Trans. Inf. Theory 51, 2859-2866 (2005)

22. Sturm, J.F.: Using SeDuMi 1.02, a MATLAB toolbox for optimization over symmetric cones. Optim. Methods Softw. 11-12, 625-653 (1999)

23. Wedderburn, J.H.M.: On hypercomplex numbers. Proc. Lond. Math. Soc. 6(2), 77-118 (1907)

24. Wedderburn, J.H.M.: Lectures on matrices, vol. 17. AMS Colloquium Publications, AMS publishers, USA (1934)

25. Zhao, Q., Karisch, S.E., Rendl, F., Wolkowicz, H.: Semidefinite Programming Relaxations for the Quadratic Assignment Problem. J. Comb. Optim. 2, 71-109 (1998) 